\title{
Design of Half-Ducted Axial Flow Fan Considering Radial Inflow and Outflow
}

\section{—Comparison of Half-Ducted Design with Ducted Design}

\author{
Pin Liu ${ }^{1}$, Yusuke Oka ${ }^{1}$, Yoichi Kinoue ${ }^{1 *}$, Norimasa Shiomi ${ }^{1}$, Toshiaki Setoguchi ${ }^{1}$, Yingzi Jin ${ }^{2}$ \\ ${ }^{1}$ Department of Mechanical Engineering, Saga University, Saga, Japan \\ ${ }^{2}$ Faculty of Mechanical Engineering \& Automation, Zhejiang Sci-Tech University, Hangzhou, China \\ Email: *kinoue@me.saga-u.ac.jp
}

Received May 27, 2013; revised June 4, 2013; accepted June 11, 2013

Copyright (C) 2013 Pin Liu et al. This is an open access article distributed under the Creative Commons Attribution License, which permits unrestricted use, distribution, and reproduction in any medium, provided the original work is properly cited.

\begin{abstract}
Half-ducted fan and ducted fan have been designed and numerically analyzed for investigating the radial flow effect on the overall performance and the three dimensional flow field in design. Based on quasi-three dimensional flow theory, the meridional flow was calculated by adopting the radial balance equations, while the calculation of the blade to blade flow was obtained by 2D cascade data with the correction by a potential flow theory. Two types of axial flow fan were designed. One is the full ducted case as if it was in the straight pipe and another is the half-ducted case with the radial inflow and outflow. The previous experimental results of authors were used to decide the inclinations of both the inflow and outflow. And the circular arc blade with equal thickness was adopted. The numerical results indicate that both of the designed fans can reach the specified efficiency and also the efficiency surpasses more than $11 \%$. Furthermore, the static pressure characteristic of half-ducted fan is much better than that of ducted fan. In comparison of the three dimensional internal flow of these two fans, the improvement of the flow angle at inlet and outlet, the distributions of velocity in the flow field and the pressure distributions on the blade surfaces can be achieved more successfully in accordance with the design intension on consideration of flow angle in design. The conclusion that half-ducted design with considering radial inflow and outflow is feasible and valid in comparison with ducted design for axial flow fans has been obtained at the end of the paper.
\end{abstract}

Keywords: Axial Flow Fan; Rotational Vortex; Half-Ducted Fans; Internal Flow; Numerical Analysis

\section{Introduction}

A lot of axial fans, which are small size and low pressure rise, are used in our daily life. Some common examples are a room ventilation fan, a radiator fan in car engine room, a power unit cooling fan of personal computer, and so on. Many of them are designed by the inverse design method. The inverse methods make a close link between the intention of designer and the blade geometry. Zangeneh [1] introduced an inverse method of a fully 3D compressible flow for the design of radial and mixed flow turbomachinery blades. The blade shape of an initial guess is obtained by the specified $r V_{t}$ and assuming uniform velocity. The 3D inverse design method was also applied to design vaned diffusers in centrifugal compressor and centrifugal and mixed flow impellers in order to investigate highly nonuniform exit flow and analyze

*Corresponding author. and minimize the generation of the secondary flows respectively $[2,3]$. The theory and application of a novel 3D inverse method for the design of turbomachinery blade in rotating viscous flow were systematically reported [4].

The inverse methods of axial flow fans can be divided into the free vortex design and controlled vortex design. In the controlled vortex design, the axial velocity can be nonuniform and the designed blade circulation can be able to specified nonconstant. Most of the axial flow fans are designed by the controlled vortex design. The proper blade loading distributions and reduced loss near the tip can be reached by the controlled vortex design [5]. The controlled vortex design also provides a method for the multistage machinery with a reasonable distribution of exit flow angle [6]. However, all these studies are not taking the radial velocity component into account. It is advantageous to consider the radial velocity in nonfree 
vortex design, which was investigated by three dimensional laser Doppler anemometer measurement [7].

In this paper, the radial flows at both the inflow and the outflow have been considered for the improvement of the design method. Two types of axial flow fans are designed by the controlled vortex design by specifying the constant tangential velocity both at inlet and outlet of the rotor. One type is ducted axial flow fan which is usually designed to prescribe almost uniform inflow and outflow as if it was in the straight pipe. However, many axial flow fans are not used in the straight pipe, such as the application of using in the ventilation and cooling systems without pipe. Thus it is important to take the real flow situation into account in design. Then the other type which is half-ducted axial flow fan was designed to compare with the traditional design of ducted ones by specifying the flow angles according to the previous experimental results of authors $[8,9]$.

\section{Design and Numerical Method}

The quasi three-dimensional flow theory was applied to investigate the flow of the axial flow fans. The meridional flow and the revolutional flow between blades were calculated by the method of streamline curvature. Based on the theory, the meridional flow was calculated by adopting the radial balance equations [10], while the calculation of the blade to blade flow was obtained by 2D cascade data with the correction by a potential flow theory so as to consider the axial flow velocity change and the inclination of the flow surface [11].

In calculation of meridional flow, the following force balance equation was evaluated at the quasi-orthogonal direction on meridional plane:

$$
\frac{\mathrm{d} V_{m}^{2}}{\mathrm{~d} q}+A(q) \cdot V_{m}^{2}=B(q)
$$

when the compressibility of the fluid is ignored,

$$
\begin{aligned}
& A(q)=2 \cdot\left\{\frac{1}{r_{m} \cdot \cos \varepsilon}+\frac{\sin \varphi \cdot \sin \varepsilon}{r}+\frac{\mathrm{d} \phi}{\mathrm{d} q} \cdot \tan \varepsilon\right\} \\
& B(q)=2 \cdot\left\{\frac{1}{\rho} \frac{\mathrm{d} P_{t}}{\mathrm{~d} q}-\frac{V_{t}}{r} \frac{\mathrm{d}\left(r V_{t}\right)}{\mathrm{d} q}\right\} \\
& V_{m}^{2}= \\
& \exp \left\{-\int A(q) \mathrm{d} q\right\}\left[\int B(q) \cdot \exp \left\{-\int A(q) \mathrm{d} q\right\}+C_{i}\right]
\end{aligned}
$$

The arbitrary constant $C_{i}$ can be got by the relative equation of mass flow rate and velocity. The energy per second getting through outlet of the rotor can be calculated by the following equation on assumption of constant $V_{t}$ at inlet and outlet ( 0 at inlet):

$$
E_{2}=\frac{\dot{m}}{K_{B}} \Delta I_{t h}=\int_{q_{h}}^{q_{c}} \rho \cdot r_{2} \cdot V_{m 2} \cdot \cos \varepsilon \cdot u_{2} \cdot V_{t 2} \mathrm{~d} q
$$

So that $V_{12}$ can be got. The total pressure rise is presumed to be able to calculate by the Euler equation as:

$$
P_{t} \cong \Delta P=\rho \cdot \eta \cdot u_{2} \cdot V_{t 2}
$$

Therefore, the meridional velocity and the tangential velocity can be obtained so that the calculation of meridional flow is finished.

The blade profile on the revolutional plane was selected by referring to the diagram of circular arc carpet. Thus, the circular arc blade with equal thickness and quadrilateral blade on the meridional plane was adopted.

Two types of axial flow fan were designed in this paper. One is the ducted case as if it was in the straight pipe and another is the half-ducted case with the radial inflow and outflow. The blade shapes on top view were obtained showing in Figure 1 on right side. The highly twisted blades can be avoided by half ducted fan with the controlled vortex design by specifying the flow angles. For the ducted fan, the streamlines on the meridional plane are uniform and parallel to each other, as shown in Figure 1(a). While the streamlines of half-ducted fan shown in Figure 1(b) obviously differ from that of ducted fan because the inclinations of inflow and outflow are given based on the experimental data [8,9] so as to consider the effect of radial velocity component on the internal flow field. The flow angles of the streamlines are given 63,16 , 38 degrees respectively on tip streamline at inlet, on tip streamline at outlet, on hub streamline at outlet.

Table 1 shows the designed parameters of ducted fan and half-ducted fan. All these parameters are specified the same value for both of the designed fans. Besides, the blade shape near the hub and the casing is not straight line but modified into spline curve, which can be seen in Figure 7(a). The flow rate and pressure rise are represented with nondimensional form of flow coefficient and pressure rise coefficient which are defined as:

$$
\begin{aligned}
& \Phi=\frac{4 Q}{\pi\left(D_{t}^{2}-D_{h}^{2}\right) \cdot U_{t}} \\
& \psi=\frac{2 p_{s}}{\rho U_{t}^{2}}
\end{aligned}
$$

and

$$
\eta=\frac{p_{s} \cdot Q}{T \cdot \omega}
$$

The designed fan blade profile data were tackled in the commercial software for the analysis of three-dimensional flow. In order to increase the speed of the numerical simulation, the internal flow fields of the axial flow 


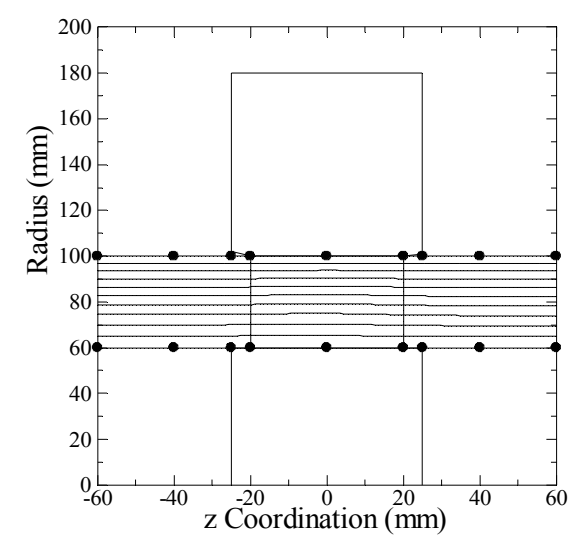

(a)

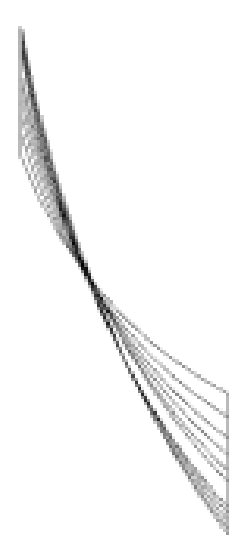

flow and top view

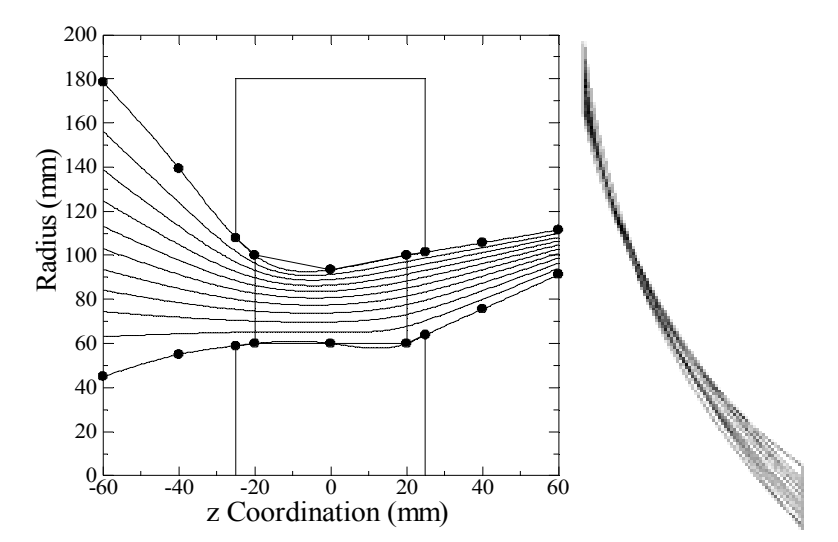

(b)

Figure 1. Outline of meridianal flow and top view of blades. (a) Ducted fan; (b) Half-ducted fan.

Table 1. Design parameters.

\begin{tabular}{lcc}
\hline & Designed Axial Flow Fan \\
\hline Tip Diameter & $D_{t}$ & $200(\mathrm{~mm})$ \\
Hub-Tip Ratio & $D_{h} / D_{t}$ & 0.6 \\
Number of Blades & $Z$ & 5 \\
Flow Coefficient & $\Phi$ & 0.264 \\
Pressure Coefficient & $\Psi$ & 0.336 \\
Rotational Speed & $n$ & $3000\left(\mathrm{~min}^{-1}\right)$ \\
Specific Speed & $N_{s}$ & $1139\left(\mathrm{~min}^{-1}, \mathrm{~m}^{3} / \mathrm{min} \mathrm{m}\right)$ \\
Efficiency & $\eta$ & $50 \%$ \\
Blockage Coefficient & $K_{B}$ & 0.96 \\
\hline
\end{tabular}

fan were divided into five periodic segments, the one fifth flow passage was numerically simulated by periodicity method with RNG $k-\varepsilon$ viscous model. The numerical calculation also take the mesh dependence into account, the mesh number between 2.06 - 3.25 million has been able to obtain the flow in general accuracy so as to the ratio of the energy obtained by the fan and the theoretical power can reach above 0.96. The pressure characteristics and the velocity field of designed halfducted and ducted fans were analyzed in the followed text.

\section{Results and Discussions}

\subsection{Aerodynamic Performance}

According to the calculation results, the efficiency computed by the Equation (9) is $62.85 \%$ and $61.2 \%$ respectively for ducted fan and half-ducted fan, both of which have completely reached to the designed value $50 \%$ and not differ so much. However, the static pressure rise of half-ducted fan is much larger than that of ducted fan, and the difference value between them can reach to 22.75 $\mathrm{Pa}$, which is relatively substantial pressure rise for a fan with a diameter of $200 \mathrm{~mm}$. So it can be said that the design of half-ducted fan which is considering the radial velocity inflow and outflow is much better than the design of the ducted fan. In addition to make this assertion amenable, the three-dimensional flow field of half-ducted fan will be described in comparison with that of ducted fan.

The following text will analyze the velocity and pressure distributions of internal flow in these two axial flow fans. The discrepancy of flow field caused by these two design method will be clarified by the numerical analysis.

\subsection{Velocity Field at Fan Inlet and Outlet}

Figures 2 and $\mathbf{3}$ present the distributions of meridional and tangential velocity at inlet and outlet of the rotor respectively for half-ducted fan and ducted fan along the radial direction. The circled lines illustrate the designed value of the tangential velocity and the lines with diamonds denote the calculated results obtained from the circumferentially averaged velocity. The meridional velocity of ducted fan is nearly uniform both at inlet and outlet, however, it is so changeable for the calculated results shown in Figure 2. The meridional velocity for half-ducted fan also doesn't come close so well, the calculated ones are lower than the designed value but they are almost in the same tendency. While the tangential velocity of half-ducted fan in calculation is much closer to designed data both at inlet and at outlet than that of the ducted fan, as shown in Figure 3. The deviations of the tangential velocity at inlet in Figure 3(a) are 2.28, 1.01, and the ones at outlet are 3.21, 2.67 in Figure 3(b), respectively for the ducted fan and half-ducted fan. The tangential velocity especially at outlet has a significant effect on pressure rise according to Euler equation as 


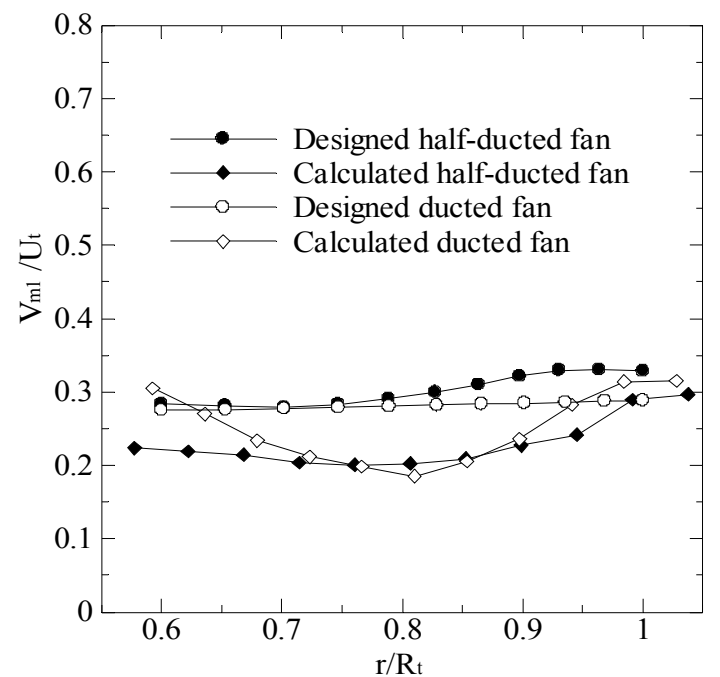

(a)

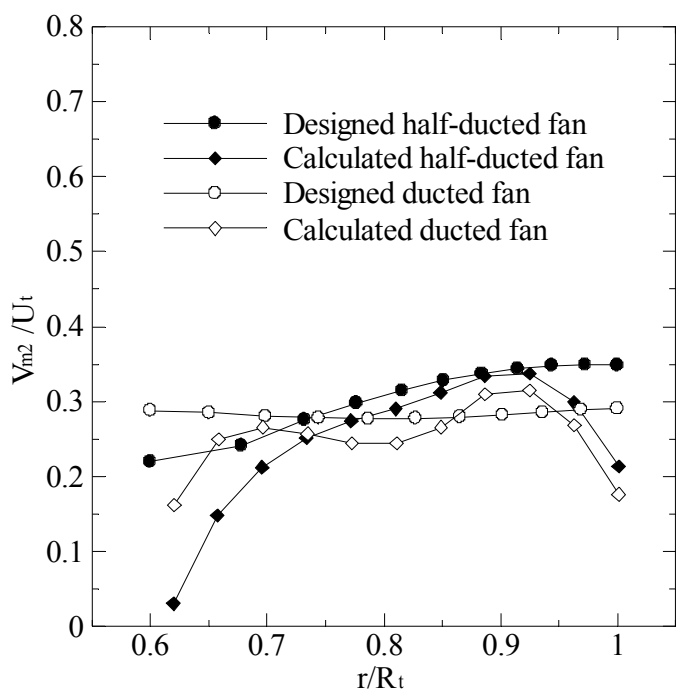

(b)

Figure 2. Circumferentially averaged velocity on meridional plane. (a) At inlet; (b) At outlet.

shown in the Equation (6).

Why the tangential velocity is so divergent from the designed data? In order to clarify the cause, the distributions of flow angles which are the intersection angle of relative velocity and meridional plane at inlet and outlet are investigated as shown in Figure 4. The lines with hollow circles and diamonds present the distributions of the inlet flow angle $\beta_{1}$ and the ones with solid circles and diamonds show the distributions of the outlet flow angle $\beta_{2}$. The deviations of the flow angles at inlet and at outlet are below 4.5 degree except the points near hub and casing at outlet. Thus the divergency of the tangential velocity of the half-ducted fan to the designed data at inlet and outlet is smaller, especially at inlet the deviation is 1.01 . However, the situation is a bit difference for the ducted

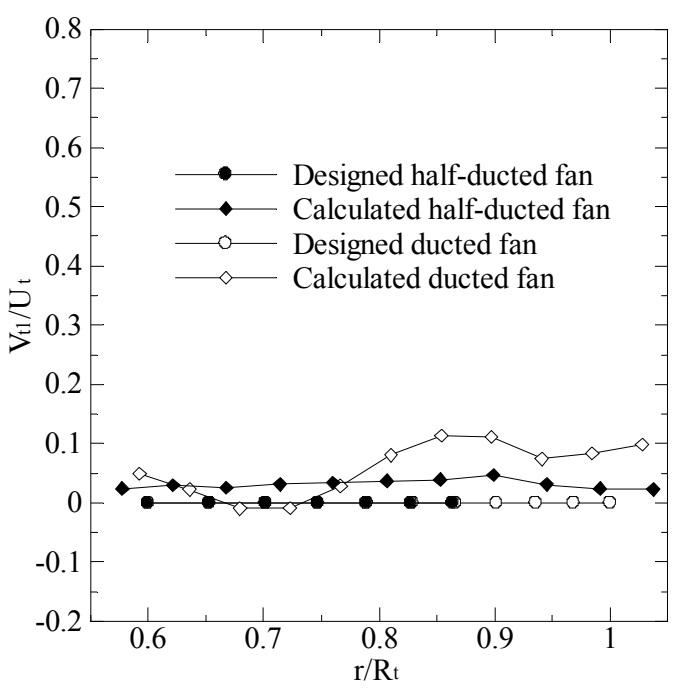

(a)

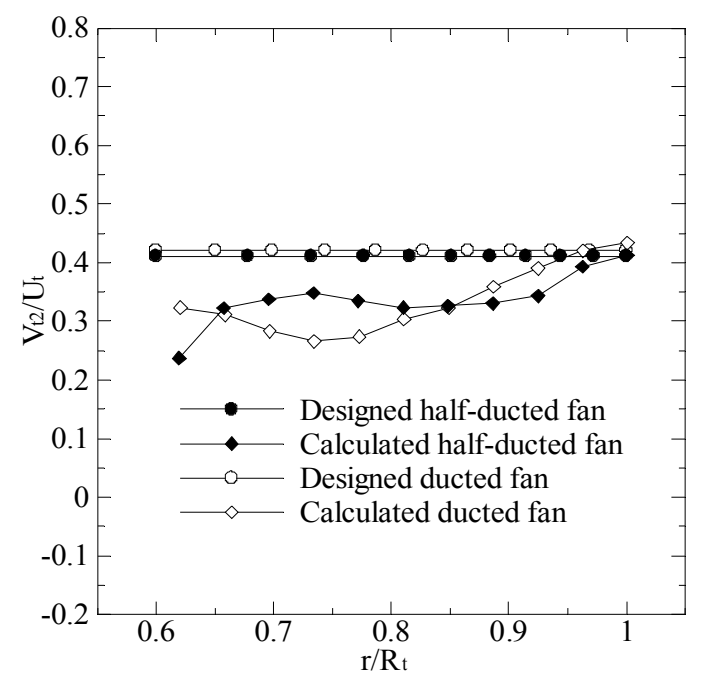

(b)

Figure 3. Circumferentially averaged tangential velocity component. (a) At inlet; (b) At outlet.

fan, the inlet flow angles does not differ so much from the designed condition of uniformed axial inflow as seen in Figure 4(b), which improves the outflow angles near the hub. However, in the dominate flow region the outflow angles are divergent from designed data, which also make tangential velocity at outlet have the deviation of 3.21 .

Figures 5 and $\mathbf{6}$ present the distributions of velocity vector on the meridional plane and a section $1 \mathrm{~mm}$ away from the blade leading edge. The meridional plane is set between blade and blade, and it locates near the leading edge of pressure surface and the trailing edge of next suction surface. The inflow of half-ducted fan is more uniform than that of the ducted fan. The vortex marked by arrows in Figures 5(b) and $\mathbf{6}$ are brought out at inlet 


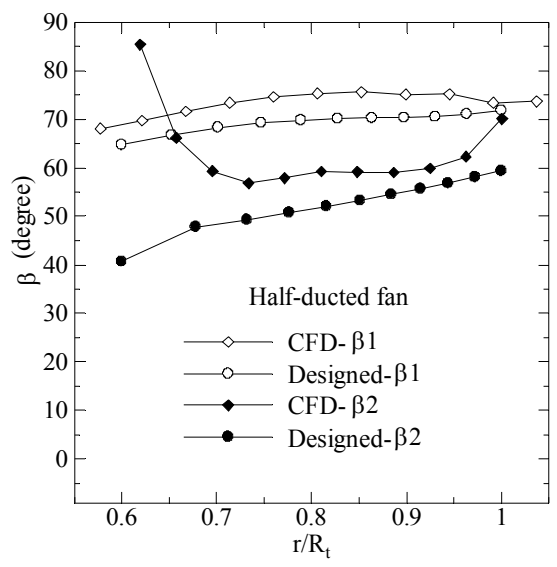

(a)

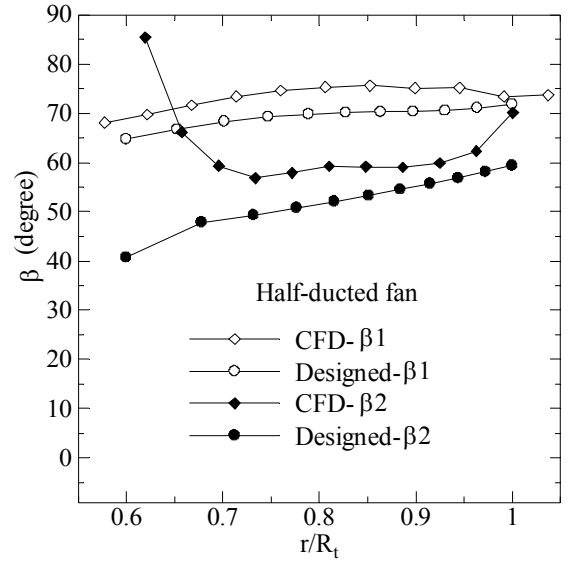

(b)

Figure 4. Distributions of flow angles. (a) Half-ducted fan; (b) Ducted fan.
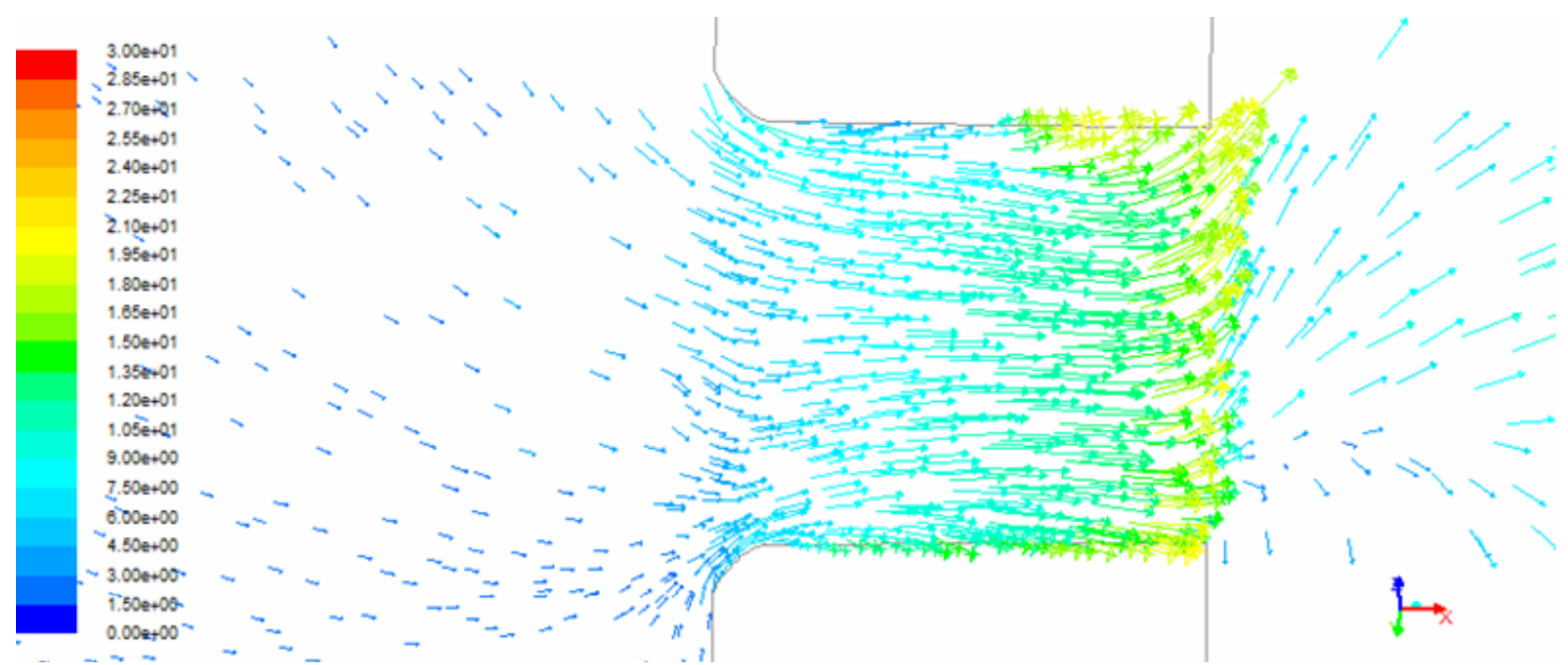

(a)
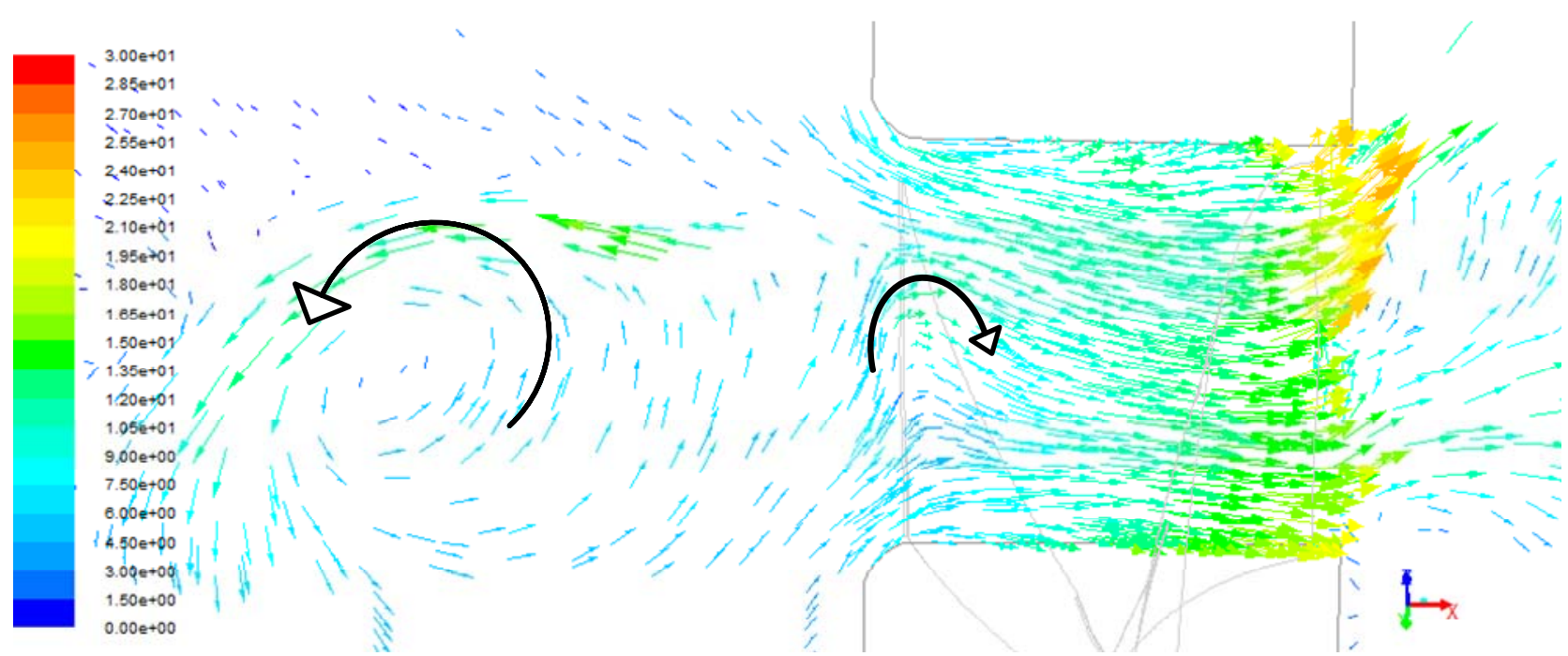

(b)

Figure 5. Distributions of velocity vector on meridional plane. (a) Half-ducted fan; (b) Ducted fan. 


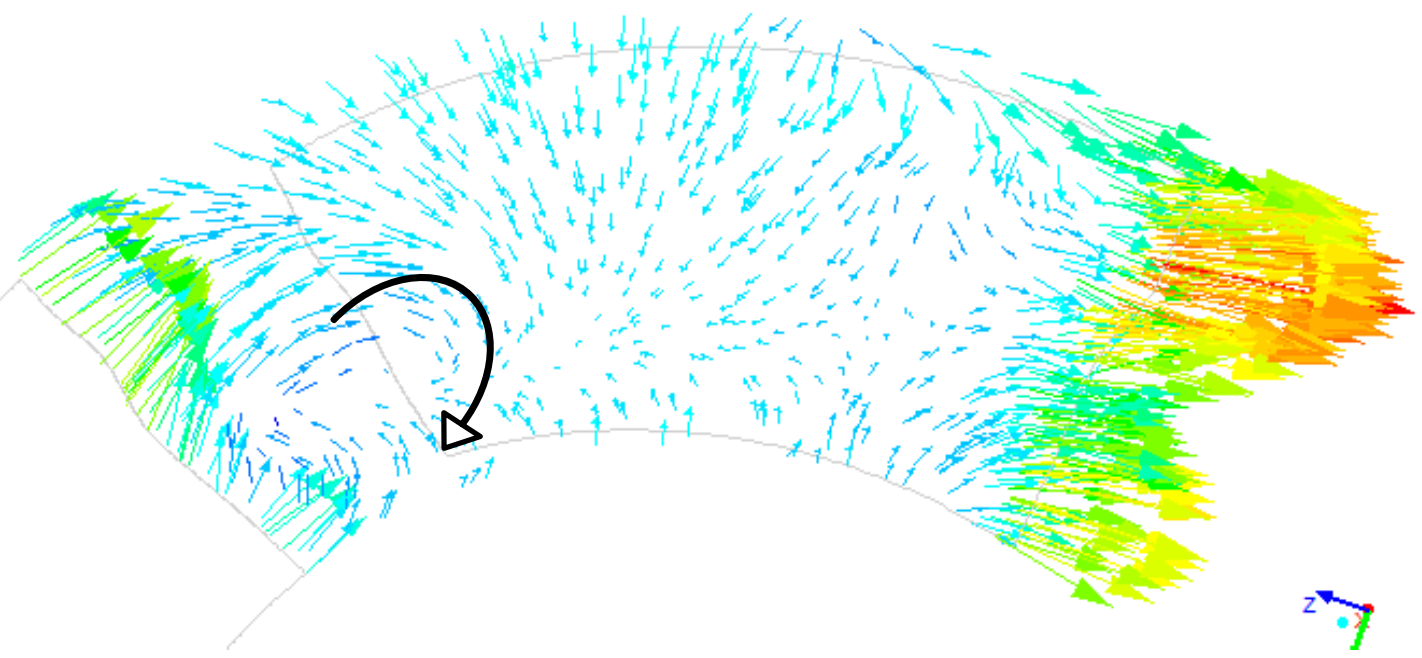

Figure 6. Distributions of velocity vector on inlet section plane (ducted fan).

of ducted fan, which cause the meridional velocity to decrease as shown in Figure 2(a) and the tangential velocity to increase as shown in Figure 3(a).

\subsection{Pressure Distributions}

Figures $\mathbf{7}$ and 8 show the distributions of static pressure on the suction surface and the pressure surface respectively. The static pressure on suction surface is negative pressure and increases from the leading edge to the trailing edge. However, the static pressure on suction surface of ducted fan increases from the mid part to the leading edge and suddenly forms a high pressure center near the tip as shown in Figure 7(b). Furthermore, the static pressure on pressure surface of ducted fan in Figure 8(b) decreases to negative near the leading edge. These phenomena observed in ducted fan weaken the suction performance of suction surface and make the flow twist at inlet region as seen in Figures 5(b) and 6. While the performance of half-ducted fan is better than that of ducted fan and the static pressure uniformly increases on pressure surface with respect to the increment of radius which follows the assumption in design well in Equation (6).

Figure 9 presents circumferentially averaged total pressure of half-ducted fan and ducted fan at outlet along radial direction. The total pressure of half-ducted fan is larger than that of ducted fan except in the casing areas and near the hub region. Furthermore, the meridional velocity dominating the flow field at outlet makes the mid-span region the most important part of the flow as seen in Figure 2(b), which is beneficial to the energy acquisition. Therefore, the static pressure rise of halfducted fan would be able to surpass that of ducted fan even they are designed by specifying the same flow parameters.

\section{Conclusions}

Half-ducted fan and ducted fan have been designed and numerically analyzed for investigating the radial flow effect on the overall performance and the three dimensional flow field in design. Higher twisted blade can be avoided by the design of half ducted fan. The numerical results indicate that both of the designed fans can reach the specified efficiency and also surpass more than $11 \%$. Furthermore, the static pressure rise of half-ducted fan is $16.6 \%$ more than that of ducted fan. In comparison of the three dimensional internal flow of these two fans, a certain number of interesting features can be summarized as follows:

1) Compared with ducted fan, the meridional velocity of half-ducted fan gets designed with one in the synchronous tendency, and the tangential velocity is much closer to designed data, which has a significant effect on the pressure rise.

2) The distributions of flow angle and velocity are improved in the dominate flow region by considering of flow angle in design of half-ducted fan.

3) The static pressure gradually increases on suction surface of half-ducted fan from leading edge to trailing edge, and also uniformly increases on pressure surface with the increment of radius in accordance with the design assumption. While in the case of ducted fan, the pressure distributions on the suction and pressure surfaces are not beneficial for the pressure rise.

As mentioned above, on consideration of flow angle in design, the improvement of the flow angle at inlet and outlet, the distributions of velocity in the flow field and 


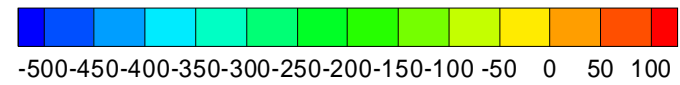

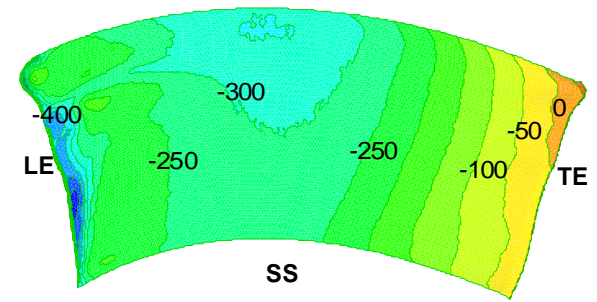

(a)

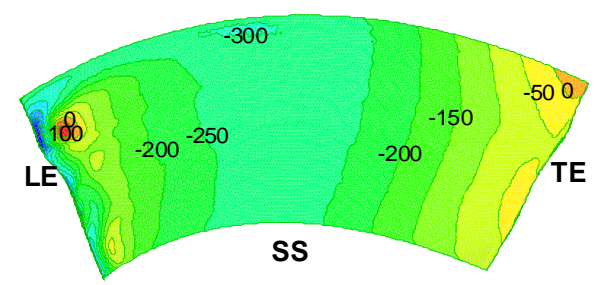

(b)

Figure 7. Distributions of static pressure on suction surface. (a) Half-ducted fan; (b) Ducted fan.

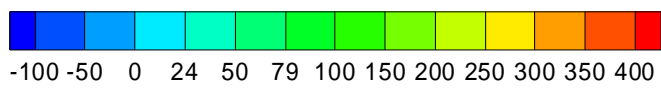

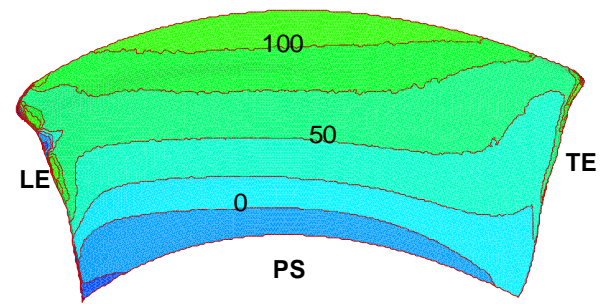

(a)

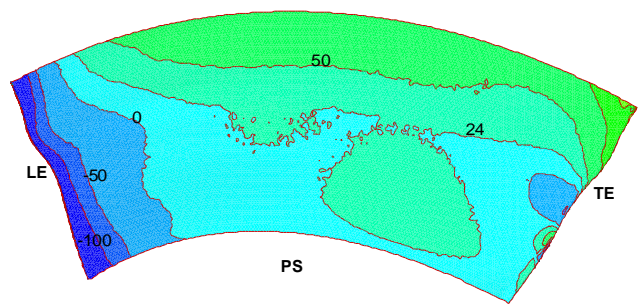

(b)

Figure 8. Distributions of static pressure on pressure surface. (a) Half-ducted fan; (b) Ducted fan.

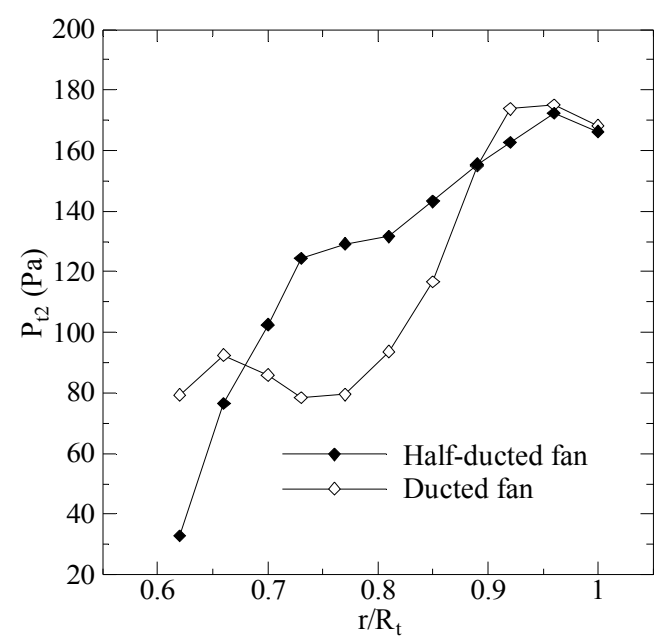

Figure 9. Total pressure at outlet.

the pressure distributions on the blade surfaces can be achieved more successfully. Therefore, half-ducted design with considering radial inflow and outflow is feasible and valid in comparison with ducted design for axial flow fans.

\section{Acknowledgements}

The authors gratefully acknowledge the research fund 2013 from the Sasagawa Scientific Research foundation.

\section{REFERENCES}

[1] M. Zangeneh, “A Compressible Three-Dimensional Design Methhod for Radial and Mixed Flow Turbomachinery Blades," International Journal of Numerical Methods in Fluids, Vol. 13, No. 5, 1991, pp. 599-624. doi:10.1002/fld.1650130505

[2] M. Zangeneh, "Inverse Design of Centrifugal Compressor Vaned Diffusers in Inlet Shear Flows," ASME Transactions on Journal of Turbomachinery, Vol. 118, No. 2, 1996, pp. 385-393. doi:10.1115/1.2836653

[3] M. Zangeneh, A. Goto and H. Harada, "On the Design Criteria for Suppression of Secondary Flows in Centrifugal and Mixed Flow Impellers," ASME Transactions on Journal of Turbomachinery, Vol. 120, No. 4, 1998, pp. 723-735. doi: $10.1115 / 1.2841783$

[4] W. T. Tiow and M. Zangeneh, "A Novel 3D Inverse Method for the Design of Turbomachinery Blades in Rotational Viscous Flow: Theory and Applications," Task Quarterly, Vol. 6, No. 1, 2002, pp. 63-78.

[5] J. Vad, A. R. A. Kwedikha, et al., "Aerodynamic Effects of Forward Blade Skew in Axial Flow Rotors of Controlled Vortex Design," Proceedings of the Institution of Mechanical Engineers, Part A: Journal of Power and Energy, Vol. 221, No. 7, 2007, pp. 1011-1023. doi:10.1243/09576509JPE420

[6] S. J. Gallimore, J. J. Bolger, et al., "The Use of Sweep and Dihedral in Multistage Axial Flow Compressor Blading-Parts 1 and 2," ASME Transactions on Journal of 
Turbomachinery, Vol. 124, No. 4, 2002, pp. 521-541. doi:10.1115/1.1507333

[7] J. Vad and F. Bencze, "Three-Dimensional Flow in Axial Flow Fans of NonFree Vortex Design," International Journal of Heat and Fluid Flow, Vol. 19, No. 6, 1998, pp. 601-607. doi:10.1016/S0142-727X(98)10004-8

[8] N. Shiomi, Y. Kinoue and T. Setoguchi, "Experimental Study on Flow Fields with Vortex in a Semi-Opened Propeller Fan," Turbomachinery, Vol. 40, No. 11, 2012, pp. 688-696.

[9] N. Shiomi, Y. Kinoue and T. Setoguchi, "Three Dimensional Velocity Fields at Rotor Outlet of a Semi-Opened
Propeller Fan," Turbomachinery, Vol. 40, No. 4, 2012, pp. 218-225.

[10] R. A. Novak, "Streamline Curvature Computing Procedures for Fluid-Flow Problems," ASME Transactions on Journal of Engineering for Power, Vol. 89, No. 2, 1967, pp. 478-490. doi:10.1115/1.3616716

[11] M. Inoue, T. Ikui, Y. Kmada and M. Tashiro, "A Quasi Three-Dimensional Design of Diagonal Flow Impellers by Use of Cascade Data," Proceeding of 10th Symposium of IAHR, Tokyo, 28 September-2 October1980, pp. 403414.

$\varepsilon$ : angle between $q$ line and normal of meridional streamline

$\eta$ : efficiency

$\rho$ : atmospheric density

$\tau$ : torque coefficient

$\Phi$ : flow coefficient

$\varphi$ : slope angle of revolution plane

$\Psi$ : pressure-rise coefficient

$\omega$ : rotating speed

\section{Subscripts}

$h$ : hub

$m$ : meridional plane

$t$ : blade tip

1/2: inlet/outlet

\section{Greek Letters}

$\beta$ : relative flow angle 